\title{
Dynamic Prediction of SARS-CoV-2 RT-PCR status on Chest Radiographs using Deep \\ Learning Enabled Radiogenomics
}

Wan Hang Keith Chiu, MBChB, FRCR ${ }^{1,2}$, Dmytro Poplavskiy $\mathrm{MSc}^{3}$, Sailong Zhang BA ${ }^{1}$, Philip Leong Ho Yu BSc, $\mathrm{PhD}^{4}$, Michael D. Kuo MD ${ }^{1,2,3 *}$

1. Department of Diagnostic Radiology, LKS Faculty of Medicine, The University of Hong Kong, Hong Kong SAR

2. Medical Artificial Intelligence Laboratory Program, Department of Diagnostic Radiology, The University of Hong Kong, Hong Kong SAR

3. Ensemblehealth.ai, Scottsdale, AZ, USA

4. Department of Mathematics and Information Technology, The Education University of Hong Kong SAR

\section{*Corresponding Author:}

Dr. Michael D. Kuo M.D.

Medical Artificial Intelligence Laboratory Program (MAIL)

Department of Diagnostic Radiology

LKS Faculty of Medicine

The University of Hong Kong

Email: mikedkuo@gmail.com

\section{Brief Introduction}

Reverse Transcription-Polymerase Chain Reaction (RT-PCR) is the gold standard for diagnosis of SARS-CoV-2 infection ${ }^{1}$, but requires specialized equipment and reagents and suffers from long turnaround times. While valuable, chest imaging currently only detects COVID-19 pneumonia, but if it can predict actual RT-PCR SARS-CoV-2 status is unknown. Radiogenomics may provide an effective and accurate RT-PCR-based surrogate ${ }^{2}$. We describe a deep learning radiogenomics (DLR) model (RadGen) that predicts a patient's RTPCR SARS-CoV-2 status solely from their frontal chest radiograph (CXR).

\section{Methods}


medRxiv preprint doi: https://doi.org/10.1101/2021.01.10.21249370; this version posted January 15, 2021. The copyright holder for this preprint (which was not certified by peer review) is the author/funder, who has granted medRxiv a license to display the preprint in perpetuity.

All rights reserved. No reuse allowed without permission.

The RadGen architecture, based on SE-ResNeXt-50-32x4d, was pretrained on ImageNet and ChestX-ray14 and 28,430 CXR from PadChest, and Kaggle before fine-tuned using CXR from a multinational cohort of RT-PCR tested patients from Hong Kong, GITHUB, SIRM and BIMCV $(6,326 \text { images })^{3-5}$. The model first predicted and selected only frontal CXR images, then predicted a segmentation mask of the cropped lung areas to reduce model fitting to unrelated parts of the image before using the segmented area as input for the RT-PCR SARS-CoV-2 binary classification task. The final prediction score was an ensemble consisting of the average of 4 models.

\section{Results}

RadGen achieved a mean Area Under the ROC curve (AUROC) of 0.959 (95\%CI $0.955,0.962)$, sensitivity of $80.8 \%(3007 / 3723)$ and specificity $95.1 \%(16206 / 17033)$ using a pre-determined 0.4 cutpoint. It distinguishes pre-COVID-19, laboratory confirmed pneumonia from SARS2-CoV-2 cases with a specificity of $89.3 \%(225 / 252)$, and a specificity of 96.4\% (106/110) on excluding SARS-CoV-2 infection on patients pre-COVID-19 CXR who later became RT-PCR positive.

The RT-PCR-tested cohort in Hong Kong consisted of 314 positive and 2,471 negative patients from 4 hospitals $^{3}$. The SARS-CoV-2 positive patients had a median of 9 (range 3-24) RT-PCR tests and 3 corresponding (range 1-8) CXR during their hospitalization (range 3-112 days); $90.8 \%$ (285/314) had mild/asymptomatic disease. The sensitivity and specificity of RadGen for predicting SARS-CoV-2 infection on initial presenting CXR was $79.5 \%$ $(225 / 283)$ and $85.2 \%(2105 / 2471)$.

RadGen time course analysis by autocorrelation function (ACF) plot, which describes how well RadGen predicts a patient's RT-PCR SARS-CoV-2 status over the course of their entire 
SARS-CoV-2 infection period, was performed revealing a peak lag at 2 days for radiogenomic signature manifestation on CXR after initial RT-PCR diagnosis. The per-film false negative rate was $10.0 \%(26 / 261)$ and $9.3 \%(21 / 225)$ within and after 7 days of the first RT-PCR positive test with a false positive rate of $68.1 \%$ (32/47) and $11.3 \%$ (45/397) within and after one week of achieving RT-PCR confirmed viral clearance (Fig 1).

\section{Comment}

Leveraging a DLR strategy and a rich body of training datasets including Asian and Western countries (reflecting a diverse set of clinical containment protocols), a wide spectrum of clinical presentations including mild and asymptomatic disease, and a prospectively collected multi-timepoint RT-PCR SARS-CoV-2 positive patient cohort, we generated a DLR model capable of predicting a patient's RT-PCR status from CXR.

Interestingly, we also show that RadGen can non-invasively 'track' RT-PCR SARS-CoV-2 status over the course of their infection, from diagnosis to viral clearance. A time-delayed correlation between RadGen and RT-PCR seen at the time of initial RT-PCR positivity and at the time of achieving RT-PCR viral clearance was observed. This is not unexpected as SARS-CoV-2 genomic dosage changes have been shown to take time to accumulate and be phenotypically reflected on a cellular, organ and systems level. Further, it is known that SARS-CoV-2 RNA can persist long after active infectivity and symptom resolution ${ }^{6}$; thus, that RadGen performs this well, particularly in a mild/asymptomatic cohort, is notable.

In conclusion, the feasibility for DLR models to dynamically track RT-PCR SARS-CoV-2 changes on an individual level significantly expands the scope of radiogenomics. 


\section{References}

1. Kuo MD, Jamshidi N. Behind the Numbers: Decoding Molecular Phenotypes with Radiogenomics_-Guiding Principles and Technical Considerations. Radiology.

2014;270(2):320-5.

2. Segal E, Sirlin CB, Ooi C, Adler AS, Gollub J, Chen X, et al. Decoding global gene expression programs in liver cancer by noninvasive imaging. Nature Biotechnology. 2007;25(6):675-80.

3. Chiu WHK, Vardhanabhuti V, Poplavskiy D, Yu PLH, Du R, Yap AYH, et al.

Detection of COVID-19 Using Deep Learning Algorithms on Chest Radiographs. Journal of Thoracic Imaging. 2020;Publish Ahead of Print.

4. Iglesia la de Vayá M, Saborit JM, Montell JA, Pertusa A, Bustos A, Cazorla M, et al. BIMCV COVID-19+: a large annotated dataset of RX and CT images from COVID-19 patients2020 June 01, 2020:[arXiv:2006.01174 p.]. Available from: https://ui.adsabs.harvard.edu/abs/2020arXiv200601174I.

5. Bustos A, Pertusa A, Salinas J-M, de la Iglesia-Vayá M. PadChest: A large chest Xray image dataset with multi-label annotated reports. arXiv e-prints [Internet]. 2019 January 01, 2019:[arXiv:1901.07441 p.]. Available from: https://ui.adsabs.harvard.edu/abs/2019arXiv190107441B.

6. Cevik M, Kuppalli K, Kindrachuk J, Peiris M. Virology, transmission, and pathogenesis of SARS-CoV-2. Bmj. 2020. 
medRxiv preprint doi: https://doi.org/10.1101/2021.01.10.21249370; this version posted January 15, 2021. The copyright holder for this preprint (which was not certified by peer review) is the author/funder, who has granted medRxiv a license to display the preprint in perpetuity.

All rights reserved. No reuse allowed without permission.

\section{Figure Legend}

Fig. 1. Serial CXR of a SARS-CoV-2 RT-PCR positive male patient in his 40s. RadGen correctly predicted the RT-PCR status of the patient on CXR prior to COVID19 (preCOVID-19), at initial SARS-CoV-2 RT-PCR confirmed diagnosis (Day 0), during his infection period (Day 8) and upon achieving RT-PCR confirmed viral clearance (Day 12). 
